\title{
Borexino: geo-neutrino measurement at Gran Sasso, Italy
}

\author{
Borexino collaboration
}

Matteo Agostini ${ }^{17,8}$, Konrad Altenmüller ${ }^{17}$, Simon Appel ${ }^{17}$, Gianpaolo Bellini ${ }^{1}$, Jay Benziger ${ }^{2}$, Daniel Bick ${ }^{3}$, Giuseppe Bonfini ${ }^{4}$, David Bravo ${ }^{5}$, Barbara Caccianiga ${ }^{1}$, Frank Calaprice ${ }^{7}$, Alessio Caminata ${ }^{9}$, Paolo Cavalcante ${ }^{4}$, Alexander Chepurnov $^{10}$, Davide D’Angelo ${ }^{1}$, Stefano Davini ${ }^{11}$, Alexander Derbin ${ }^{12}$, Lea Di Noto ${ }^{9}$, Ilya Drachnev ${ }^{8}$, Alexander Etenko ${ }^{13,14}$, Kirill Fomenko ${ }^{4,15}$, Andrey Formozov ${ }^{15,1}$, Davide Franco ${ }^{16}$, Federico Gabriele ${ }^{4}$, Cristian Galbiati ${ }^{7}$, Chiara Ghiano ${ }^{9}$, Marco Giammarchi ${ }^{1}$, Marianne Göger-Neff ${ }^{17}$, Augusto Goretti ${ }^{7}$, Maxim Gromov ${ }^{10}$, Caren Hagner ${ }^{3}$, Ed Hungerford ${ }^{11}$, Aldo Ianni ${ }^{4}$, Andrea Ianni ${ }^{7}$, Karol Jedrzejczak ${ }^{21}$, Dominik Jeschke ${ }^{17}$, Vladislav Kobychev ${ }^{18}$, Denis Korablev ${ }^{15}$, Gyorgy Korga ${ }^{11}$, Didier Kryn ${ }^{16}$, Matthias Laubenstein ${ }^{4}$, Bjoern Lehnert ${ }^{19}$, Evgeny Litvinovich ${ }^{13,14}$, Francesco Lombardi ${ }^{4}$, Paolo Lombardi ${ }^{1}$, Livia Ludhova ${ }^{1}$, Georgy Lukyanchenko ${ }^{13}$, Igor Machulin ${ }^{13,14}$, Szymon Manecki ${ }^{5}$, Werner Maneschg ${ }^{20}$, Simone Marcocci ${ }^{8,9}$, Emanuela Meroni ${ }^{1}$, Mikko Meyer ${ }^{3}$, Lino Miramonti ${ }^{1}$, Marcin Misiaszek ${ }^{21}$, Michele Montuschi ${ }^{25}$, Pablo Mosteiro ${ }^{7}$, Valentina Muratova ${ }^{12}$, Birgit Neumair ${ }^{17}$, Lothar Oberauer ${ }^{17}$, Michel Obolensky ${ }^{16}$, Fausto Ortica ${ }^{22}$, Marco Pallavicini ${ }^{9}$, Laszlo Papp ${ }^{5,17}$, Laura Perasso ${ }^{9}$, Andrea Pocar ${ }^{6}$, Gioacchino Ranucci ${ }^{1{ }^{\star}}$, Alessandro Razeto ${ }^{4}$, Alessandra $\operatorname{Re}^{1}$, Aldo Romani ${ }^{22}$, Romain Roncin ${ }^{4,16}$, Nicola Rossi ${ }^{4}$, Stefan Schönert ${ }^{17}$, Dmitrii Semenov ${ }^{12}$, Hardy Simgen ${ }^{20}$, Mikhail Skorokhvatov ${ }^{13,14}$, Oleg Smirnov ${ }^{15}$, Albert Sotnikov ${ }^{15}$, Serguei Sukhotin ${ }^{13}$, Yury Suvorov ${ }^{4,13,23}$, Roberto Tartaglia ${ }^{4}$, Gemma Testera ${ }^{9}$, Jan Thurn ${ }^{19}$, Maria Toropova ${ }^{13}$, Evgenii Unzhakov ${ }^{12}$, Alina Vishneva ${ }^{15}$, Robert Bruce Vogelaar ${ }^{5}$, Franz von Feilitzsch ${ }^{17}$, Hanguo Wang ${ }^{23}$, Stefan Weinz ${ }^{24}$, Juergen Winter ${ }^{24}$, Marcin Wojcik ${ }^{21}$, Michael Wurm ${ }^{24}$, Zachary Yokley ${ }^{5}$, Oleg Zaimidoroga ${ }^{15}$, Sandra Zavatarelli ${ }^{9}$, Kai Zuber ${ }^{19}$, Grzegorz Zuzel ${ }^{21}$

\footnotetext{
${ }^{1}$ Università di Milano, Dipartimento di Fisica, and INFN, Milan, Italy; ${ }^{2}$ Princeton University, Chemical Engineering Department, Princeton, New Jersey, USA; ${ }^{3}$ Institut für Experimentalphysik, Universität Hamburg, Hamburg, Germany; ${ }^{4}$ Istituto Nazionale di Fisica Nucleare (INFN), Laboratori Nazionali del Gran Sasso, Assergi (L'Aquila), Italy; ${ }^{5}$ Virginia Polytechnic Institute and State University, Physics Department, Blacksburg, Virginia, USA; ${ }^{6}$ University of Massachusetts, Physics Department, Amherst, Massachusetts, USA; ${ }^{7}$ Princeton University, Physics Department, Princeton, New Jersey, USA; ${ }^{8}$ Gran Sasso Science Institute (INFN), L'Aquila, Italy; ${ }^{9}$ Università di Genova, Dipartimento di Fisica, and INFN, Genoa, Italy; ${ }^{10}$ Lomonosov Moscow State University Skobeltsyn Institute of Nuclear Physics, Moscow, Russia; ${ }^{11}$ University of Houston, Department of Physics, Houston, Texas, USA; ${ }^{12}$ St Petersburg Nuclear Physics Institute, Gatchina, Russia; ${ }^{13}$ NRC Kurchatov Institute, Moscow, Russia; ${ }^{14}$ National Research Nuclear University MEPhI (Moscow Engineering Physics Institute), Moscow, Russia; ${ }^{15}$ Joint Institute for Nuclear Research, Dubna, Russia; ${ }^{16}$ APC, Université Paris Diderot, CNRS/IN2P3, CEA/Irfu, Observatoire de Paris, Sorbonne Paris Cité, Paris, France; ${ }^{17}$ Technische Universität München, Physik-Department and Excellence Cluster Universe, Garching, Germany; ${ }^{18}$ Kiev Institute for Nuclear Research, Kiev, Ukraine; ${ }^{19}$ Technische Universität Dresden, Fachrichtung Physik, Dresden, Germany; ${ }^{20}$ Max-Planck-Institut für Kernphysik, Heidelberg, Germany; ${ }^{21}$ M. Smoluchowski Institute of Physics, Jagiellonian University, Krakow, Poland; ${ }^{22}$ Università di Perugia, Dipartimento di Chimica, Biologia e Biotecnologie, and INFN, Perugia, Italy; ${ }^{23}$ University of California Los Angeles (UCLA), Physics and Astronomy Department, Los Angeles, California, USA; ${ }^{24}$ Institut für Physik, Johannes Gutenberg Universität Mainz, Mainz, Germany; ${ }^{25}$ Università di Ferrara, Dipartimento di Fisica e Scienze della Terra, and INFN, Ferrara, Italy
}

\section{Article history}

Received March 11, 2016; accepted October 5, 2016.

Subject classification:

Geoneutrinos, Solar neutrinos, Neutrino oscillations, Liquid scintillators, Low background. 


\section{ABSTRACT}

Geo-neutrinos, electron anti-neutrinos produced in $\beta$-decays of naturally occurring radioactive isotopes in the Earth, are a unique direct probe of our planet's interior. After a brief introduction of the geo-neutrinos' properties and of the main aims of their study, we discuss the features of a detector which has recently provided breakthrough achievements in the field, Borexino, a massive, calorimetric liquid scintillator detector installed at the underground Gran Sasso Laboratory. With its unprecedented radiopurity levels achieved in the core of the detection medium, it is the only experiment in operation able to study in real time solar neutrino interactions in the challenging sub-MeV energy region. Its superior technical properties allowed Borexino also to provide a clean detection of terrestrial neutrinos. Therefore, the description of the characteristics of the detected geo-neutrino signal and of the corresponding geological implications are the main core of the discussion contained in this work.

\section{Introduction}

Borexino at Gran Sasso is the last player which entered the solar neutrino arena, where, thanks to its unprecedented low background, it provided breakthrough results in the low energy sub-MeV regime. Having already measured three components of the solar neutrino spectrum over the past years, i.e. ${ }^{7} \mathrm{Be},{ }^{8} \mathrm{~B}$ and pep (providing jointly with this component also a stringent upper limit on the CNO contribution), Borexino has recently crowned its remarkable series of results with the detection of the fundamental pp neutrino flux, coming from the reaction which provides most of the Sun's energy. Therefore, Borexino is the first experiment able to perform an almost complete spectroscopy of the whole solar neutrino flux, allowing a thorough data-model comparison [Serenelli et al. 2011]. The last step of this investigation will be the attempt to measure the tiny $\mathrm{CNO}$ flux, which Borexino will undertake over the next years.

Moreover, specifically relevant to the present discussion, Borexino has also detected an unquestionable geo-neutrino signal, contributing to pave the way to a complete new method to investigate the interior of our planet based on the study of the properties of such elusive messengers.

In the following, after an introduction to the underground Gran Sasso Laboratory hosting the experiment, and a general highlight of the features of geo-neutrinos from Earth, the experimental characteristics of Borexino, which made possible the outstanding accomplishments accumulated so far, will be briefly reviewed.

Afterwards, the characteristics of the geo-neutrino signal detected by Borexino will be thoroughly described and quantified, and its geological implications carefully emphasized.

Finally, the future perspective of this new and fascinating field will be sketched, pointing to the potential big advantage for the study of the interior of our planet that would stem from a network of powerful geo-neutrino detectors deployed around the world.

\section{The hosting laboratory: Laboratori Nazionali del Gran Sasso in Italy}

Since a fundamental prerequisite for the breakthrough results of Borexino is the hosting laboratory, few words for its description are mandatory.

The LNGS facility (Laboratori Nazionali del Gran Sasso; http: / / www.lngs.infn.it/) under the Gran Sasso mountain in the Abruzzo region of Italy, is currently the largest of the underground facilities all over the world. Following the proposal in 1979 by A. Zichichi, the INFN President at that time, construction was completed in 1987, together with two road tunnels through the mountain. These road tunnels provide the horizontal access by road vehicle to the facility, allowing transport container delivery.

The underground area comprises three main hallways, with several other experimental tunnels available, including two $90 \mathrm{~m}$ hallways used for a Michelson interferometer for geological studies.

The dimensions of the Hall $\mathrm{C}$ are $100 \mathrm{~m}$ by $20 \mathrm{~m}$ by $18 \mathrm{~m}$, with the other two, A and B, only slightly smaller, providing a total area of $17,300 \mathrm{~m}^{2}$, and a total volume of $180,000 \mathrm{~m}^{3}$. A surface set of auxiliary buildings provides offices and administration facilities, computing and conferencing capabilities, laboratories, electrical and mechanical workshops and warehousing facilities. There is a permanent staff of 76 physicists, engineers and technicians, with about 20 non-permanent staff, servicing about 800 users from across 26 countries.

The rock overburden is $1400 \mathrm{~m}$, with an azimuthal and zenith distribution, due to the topology of the mountain, which varies between 3100 m.w.e. and 3800 m.w.e. This reduces the muon flux to a measured value of $2.87 \times 10^{-4} / \mathrm{m}^{2} / \mathrm{s}$ [Ahlen et al. 1990, Aglietta et al. 1998]. Measured neutron fluxes are $2.93 \times 10^{2} / \mathrm{m}^{2} / \mathrm{s}$ and $0.86 \times 10^{2} / \mathrm{m}^{2} / \mathrm{s}$ for thermal and fast neutrons (with a breakpoint defined at $1 \mathrm{keV}$ ), respectively [Belli et al. 1989]. Radon levels in the air range between 50 and 120 $\mathrm{Bq} / \mathrm{m}^{3}$ with the ventilation system providing one exchange of air in $3.5 \mathrm{~h}$.

The science program at LNGS is very broad, with neutrino beam studies just completed (OPERA and ICARUS), galactic dark matter searches (DAMA/LIBRA, DarkSide, XENON, CRESST), ov $\beta \beta$ decay studies (CUORE, GERDA, LUCIFER and COBRA), solar and geo-neutrinos (Borexino, the topic of this discussion), supernova neutrinos (LVD, Borexino) and nuclear astrophysics through a low energy ion accelerator (LUNA). In addition, low background counting facilities exist, like the STELLA HPGe detector array. 
In terms of expansion of the facility, there are no current plans to extend the available underground space, with new areas becoming available as the current experimental program evolves and projects are completed.

\section{Geo-neutrinos}

Geo-neutrinos $\left(\right.$ geo- $\left.\bar{\nu}_{\mathrm{e}}\right)$ are electron anti-neutrinos $\left(\bar{v}_{\mathrm{e}}\right)$ produced in $\beta$-decays of ${ }^{40} \mathrm{~K}$ and of several nuclides in the chains of the long-lived radioactive isotopes ${ }^{238} \mathrm{U}$ and ${ }^{232} \mathrm{Th}$, which are naturally present in the Earth:

$$
\begin{gathered}
{ }^{238} \mathrm{U} \rightarrow{ }^{206} \mathrm{~Pb}+8 \alpha+8 \mathrm{e}^{-}+6 \bar{\nu}_{\mathrm{e}}+51.7 \mathrm{MeV} \\
{ }^{232} \mathrm{Th} \rightarrow{ }^{208} \mathrm{~Pb}+6 \alpha+4 \mathrm{e}^{-}+4 \bar{v}_{\mathrm{e}}+42.8 \mathrm{MeV} \\
{ }^{40} \mathrm{~K} \rightarrow{ }^{40} \mathrm{Ca}+\mathrm{e}^{-}+\bar{v}_{\mathrm{e}}+1.32 \mathrm{MeV}
\end{gathered}
$$

Hence, while the Sun shines in neutrinos, the Earth shines in geo-neutrinos, with a flux of about $10^{6} \mathrm{~cm}^{-2} \mathrm{~s}^{-1}$. It is important to note that the released radiogenic heat is in a well fixed and known proportion with respect to the geo-neutrino flux. Therefore, it is in principle possible to determine the Urey ratio (defined as the relative contribution of the radiogenic heat from radioactive decays to the Earth's surface heat flux) by measuring the geo-neutrino flux.

In particular, by determining experimentally the geo-neutrino signal at different locations through the planet, in different geological settings, and/ or by being able to identify the incoming direction of detected geoneutrinos, it might be possible to:

a) study the distribution of radioactive elements within the Earth, to determine their abundances in the crust and in the mantle;

b) determine if there are any radioactive elements in the Earth's core;

c) understand if the mantle composition is homogeneous or not;

d) test, validate, and discriminate among different BSE models;

e) exclude or confirm the presence of a putative georeactor in the core of the planet;

f) determine the Urey ratio, important for geochemistry and geophysics.

g) study the bulk $U$ and Th ratio in the silicate Earth, an important parameter for geochemistry, which could shed light on the process of the Earth's formation.

We can see, hence, that geo-neutrinos can be used as a unique direct probe of the Earth interior, not accessible by any other means. All this information could be used as inputs for many geological, geophysical, and geochemical models describing such complex processes as the mantle convection, movement of tectonic plates,

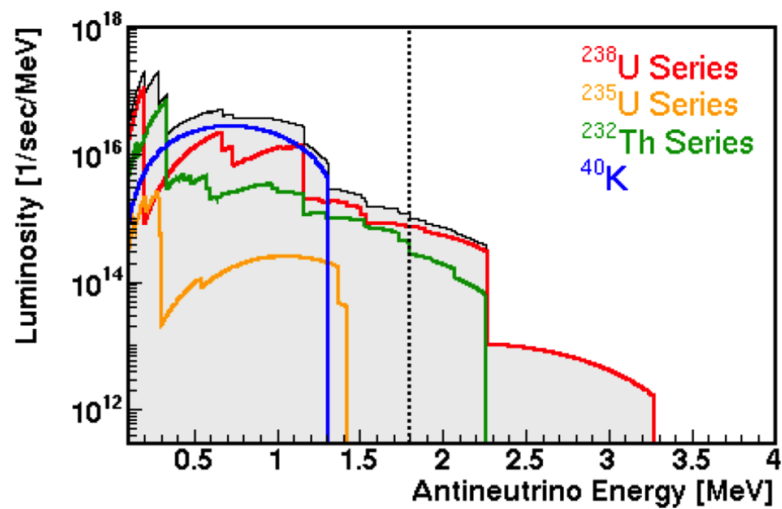

Figure 1. The geo-neutrino luminosity as a function of energy is shown for the most important reaction chains and nuclides [Enomoto 2009]. Only geo-neutrinos of energies above the $1.8 \mathrm{MeV}$ energy (vertical dashed line) can be detected by means of the inverse beta decay on target protons shown in Equation (4). For completeness, though not mentioned in the text, also the ${ }^{235} \mathrm{U}$ contribution is indicated.

geo-dynamo (the process of the generation of the Earth's magnetic field), the process of the Earth formation, etc.

The energy spectrum of geo-neutrinos extends to about $3.3 \mathrm{MeV}$. They are detected via the inverse neutron $\beta$-decay reaction

$$
\bar{v}_{\mathrm{e}}+p \rightarrow e^{+}+n
$$

which has a kinematic threshold of $1.806 \mathrm{MeV}$. The cross section of this interaction as a function of anti-neutrino energy is well known and can be found in Strumia and Vissani [2003]. Unfortunately, all geo-neutrinos produced in the decay of ${ }^{40} \mathrm{~K}$ are below this threshold and we are able to detect only the tail of the ${ }^{238} \mathrm{U}$ and ${ }^{232} \mathrm{Th}$ geo-neutrinos, as shown by the dashed line in Figure 1 . Geo-neutrinos from the ${ }^{232} \mathrm{Th}$ chain have the end point of their energy spectrum at about $2.25 \mathrm{MeV}$, while those from the ${ }^{238} \mathrm{U}$ chain extend up to $3.3 \mathrm{MeV}$. Ideally, this spectral feature could be used in order to measure the $\mathrm{U}$ and Th ratio in the Earth. It is important to recall that the relative proportions of the elements abundances are much better known than their absolute abundances. Therefore, by measuring the absolute abundances of ${ }^{238} \mathrm{U}$ and ${ }^{232} \mathrm{Th}$, the absolute abundance of ${ }^{40} \mathrm{~K}$ can be deduced with a better precision.

Geo-neutrinos are emitted and interact as flavor states, but they travel as superposition of mass states and therefore undergo flavor oscillations.

In the approximation $\Delta m_{31}^{2} \sim \Delta m_{32}^{2}>>\Delta m_{21}^{2}$ (the square-mass differences of mass eigenstates $1,2,3$ ) the survival probability $P_{e e}$ for a $\bar{v}_{\mathrm{e}}$ in vacuum is:

$$
\begin{gathered}
P_{e e}=P\left(\bar{v}_{\mathrm{e}} \rightarrow \bar{v}_{\mathrm{e}}\right)=\sin ^{4} \theta_{13}+ \\
+\cos ^{4} \theta_{13}\left(1-\sin ^{2} 2 \theta_{12} \sin ^{2}\left(\frac{1.267 \Delta m_{21}^{2}\left[e V^{2} L[m]\right.}{4 E[M e V]}\right)\right)
\end{gathered}
$$


In the Earth, the geo-neutrino sources span a vast region compared to the oscillation length, given by:

$$
L \approx 2.48 m \frac{E[\mathrm{MeV}]}{\Delta m_{21}^{2}\left[e V^{2}\right]}
$$

For example, for a $\sim 3 \mathrm{MeV}$ antineutrino, the oscillation length is of $\sim 100 \mathrm{~km}$, small with respect to the Earth's radius of $\sim 6371 \mathrm{~km}$ : the effect of the neutrino oscillation to the total neutrino flux is, therefore, completely averaged, giving an overall survival probability of:

$$
\left\langle P_{e e}\right\rangle \approx \cos ^{4} \theta_{13}\left[1-\frac{1}{2} \sin ^{2} 2 \theta_{12}\right]+\sin ^{4} \theta_{13}
$$

According to the neutrino oscillation mixing angles and square-mass differences reported in Fogli et al. [2012], $P_{e e} \sim 0$.54. It has been calculated in Enomoto [2005] that the so called matter effect contribution to the average survival probability is an increase of about $2 \%$ and the spectral distortion is below $1 \%$. To conclude, the net effect of flavor oscillations during the geo-neutrinos propagation through the Earth is an absolute decrease of the overall flux by $\sim 0.55$, with a very small spectral distortion, negligible for the precision of the current geo-neutrino experiments.

\section{Characteristics of the Borexino experiment}

Borexino [Alimonti et al. 2009] is a scintillator detector, which employs as active detection medium 300 tons of pseudocumene-based scintillator. The intrinsic high luminosity of the liquid scintillation technology is the key toward the initial design goal of Borexino, the real time observation of sub-MeV solar neutrinos through $v e$ elastic scattering, being the ${ }^{7} \mathrm{Be}$ component the main foreseen target at the time of the proposal. However, the lack of directionality of the method makes it impossible to distinguish neutrino-scattered electrons from electrons due to natural radioactivity, thus leading to the other crucial requirement of the Borexino technology, e.g. an extremely low radioactive contamination of the detection medium, to a degree never reached before. Specifically, $10^{-16} \mathrm{gs} / \mathrm{g}$ of ${ }^{238} \mathrm{U}$ and ${ }^{232} \mathrm{Th}$, and $10^{-18} \mathrm{gs} / \mathrm{g}$ of ${ }^{40} \mathrm{~K}$ were the initial radiopurity specifications of the experiment.

The active scintillating volume is observed by 2212 PMTs located on a $13.7 \mathrm{~m}$ diameter sphere and is shielded from the external radiation by more than 2500 tons of water and by 1000 tons of hydrocarbon equal to the main compound of the scintillator (pseudocumene), to ensure zero buoyancy on the thin Nylon Inner Vessel containing the scintillator itself. Key factors for the accomplishment of the planned measurements of the

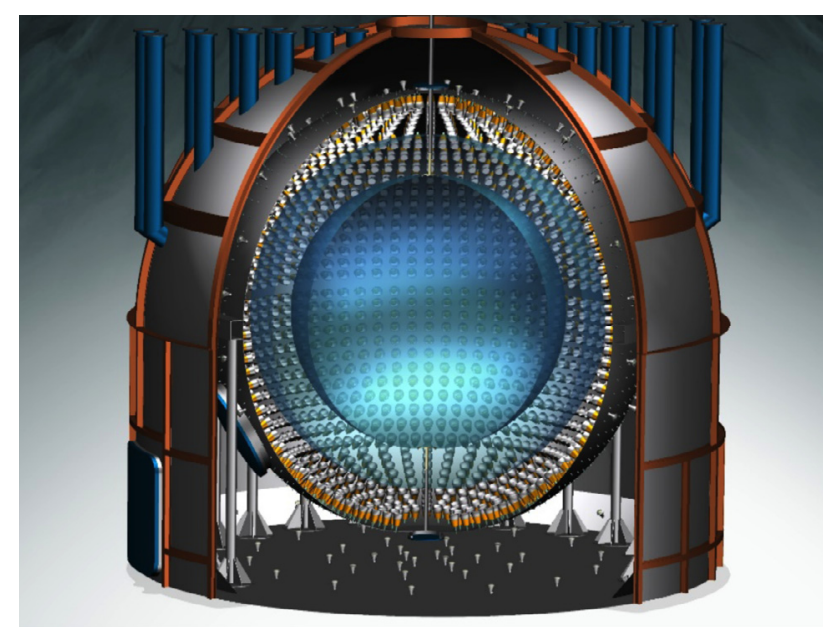

Figure 2. Schematic pictorial view of the Borexino detector.

experiment were also the purification and handling systems, designed and installed to ensure the manipulation and decontamination of the fluids at the radiopurity levels mentioned above [Bellini et al. 2009].

When data taking started in May 2007, it appeared immediately that the task of the ultralow radioactivity was successfully obtained, with residual contaminations even better than the design values ( $\mathrm{U}$ and $\mathrm{Th}$ at $10^{-18} \mathrm{gs} / \mathrm{g}$ and ${ }^{40} \mathrm{~K}$ not detectable), representing per se a major technological breakthrough, opening a new era in the field of ultrapure detectors for rare events search. The achieved ultra-low background implies that, once selected by software analysis the design fiducial volume of 100 tons and upon removal of the muon and muoninduced signals, the recorded experimental spectrum is clean enough to show the feature of the ${ }^{7} \mathrm{Be}$ scattering edge, i.e. the unambiguous signature of the occurrence of solar neutrino detection [Bellini et al. 2011].

As additional bonus, the better-than-anticipated features manifested by the detector made it also clear since the beginning of the physics run that the geo neutrino detection would have been amply within the reach of Borexino, as later on confirmed after the accumulation of enough data statistics.

An overall pictorial view of the detector is reported in Figure 2.

\subsection{Detector response}

Several steps are required to extract from the raw data the quantitative information of interest regarding the solar neutrino and geo-neutrino fluxes. The raw signals must be converted into meaningful amplitude variables; of the total accumulated signals only those satisfying the scintillation event acceptance criteria are kept; the data spectrum is constructed by including only events which are reconstructed within a fiducial volume far from the wall of the containment vessel; and, finally, the spectrum is fitted to a global signal-plus- 
background model in which the flux amplitude to be evaluated is a fit parameter.

The task to obtain a detailed understanding of the detector response has been accomplished through two coordinated efforts, i.e. a thorough calibration campaign and the development of a full MC able to reproduce the detector performances.

\subsection{Calibration}

The calibration of the detector has been accomplished to characterize both the energy and time response of Borexino. Several sources have been deployed in different locations within the liquid scintillator: gamma sources producing monoenergetic lines spanning the energy range of interest from 0.122 to $1.4 \mathrm{MeV}$, a Radon source realized by filling with liquid scintillator taken from the Inner Vessel a small quartz vial which was successively loaded with Radon, and an Am-Be neutron source, specifically employed to test and characterize the anti-neutrino detection process (see previous Equation (4)).

The gamma lines and the Radon source, positioned in several hundred locations, gave an accurate probe of the energy response of the experiment as function of the event position; furthermore they also provided a way to careful calibrate the capability of the time signals from the array of photomultipliers to identify precisely the vertex of the events.

Jointly, the energy and spatial measurements obtained throughout the calibration campaign provided a complete map of the detector response, leading to the following estimates of the crucial features of the detector: energy resolution $5 \% / \sqrt{ } \mathrm{E}(\mathrm{MeV})$, linearity of the energy scale of the order of $1 \%$, fiducial volume uncertainty close to $1 \%$, too [Back et al. 2012].

\subsection{MC tuning}

The second ingredient at the basis of the precise measurements accomplished is the accurate Monte Carlo description of the detector. It required a while to develop a full MC code incorporating all the details of the light generation and transport in the liquid scintillator, of the behavior of the photomultipliers and of the electronics response. At the end of this development, we were able to produce a full simulation suited to be confronted with the many outputs stemming from the calibration campaign.

Such a comparison has been used to contrast the source data with the corresponding simulated events; in this way it has been possible on one hand to perform a fine tuning of the MC code, so to ensure the best match between the measured and simulated data, and on the other to quantify precisely the crucial, residual uncertainties on the energy scale and on the fiducial volume, already listed before. A thorough account of the effort to characterize the performances of the detector has been published in Bellini et al. [2014], where also the full methodology adopted for the solar neutrino analysis is described.

\section{Geo-neutrino results}

The Borexino geo-neutrino result [Bellini et al. 2013] reported here refers to the statistics collected from December 2007 to August 2012, corresponding to 1352.60 days of live time. The corresponding fiducial exposure after all cuts is $(613 \pm 26)$ ton $\times$ year or $(3.69 \pm 0.16) \times 10^{31}$ proton $\times$ year.

Electron anti-neutrinos are measured through the inverse neutron $\beta$-decay reaction of Equation (4). The positron created in this reaction promptly comes to rest and annihilates. All deposited energy is detected in a single prompt event, with a visible energy of $E_{\text {prompt }}=$ $E_{\bar{v}_{\mathrm{e}}}-0.784 \mathrm{MeV}$. The emitted free neutron is typically captured on protons, resulting in the emission of a 2.22 $\mathrm{MeV}$ de-excitation $\gamma$ ray, providing a delayed coincidence event. The mean neutron-capture time in Borexino was measured with an AmBe neutron source, resulting equal to $\tau=(254.5 \pm 1.8) \mu$ s. The characteristic time and spatial coincidence of prompt and delayed events offers a clean signature of $\bar{v}_{\mathrm{e}}$ detection, further suppressing possible background sources.

The $\bar{v}_{\mathrm{e}}$ 's from nuclear power plants are the main anti-neutrino background to the geo-neutrino measurement. Since there are no near-by nuclear plants, the LNGS site is well suited for geo-neutrino detection. The number of expected $\bar{v}_{\mathrm{e}}$ reactor candidates is $N_{\text {react }}=$ $(33.3 \pm 2.4)$ events, after cuts. Thanks to the extreme radiopurity of the Borexino detector, the non anti-neutrino background is almost negligible, only $0.7 \pm 0.18$ events, dominated by $\beta+$ neutron decays of cosmogenic ${ }^{9} \mathrm{Li}$ and ${ }^{8} \mathrm{He}$, accidental coincidences, and $(\alpha, n)$ reactions with $\alpha$ 's from decays of ${ }^{210} \mathrm{Po}$.

Through the application of the selection cuts, in the period under consideration 46 antineutrino candidates have been selected, whose prompt energy spectr (i.e. the spectrum of the positron in Equation (4)) is shown in Figure 3. An unbinned maximal likelihood fit of the energy spectrum (expressed in unit of photoelectrons) of the prompt candidates was performed, with the $\mathrm{Th} / \mathrm{U}$ mass ratio fixed to the chondritic value of 3.9, and with the number of events from reactor antineutrinos left as a free parameter.

The resulting best fit values are $N_{\text {geo }}=(14.3 \pm 4.4)$ geo-neutrino events, and $N_{\text {react }}=31.2_{-6.1}^{+7.0}$ reactor events. If converted to the standard unit used in this field, the TNU (defined as 1 TNU $=1$ Terrestrial Neutrino Unit $=$ 


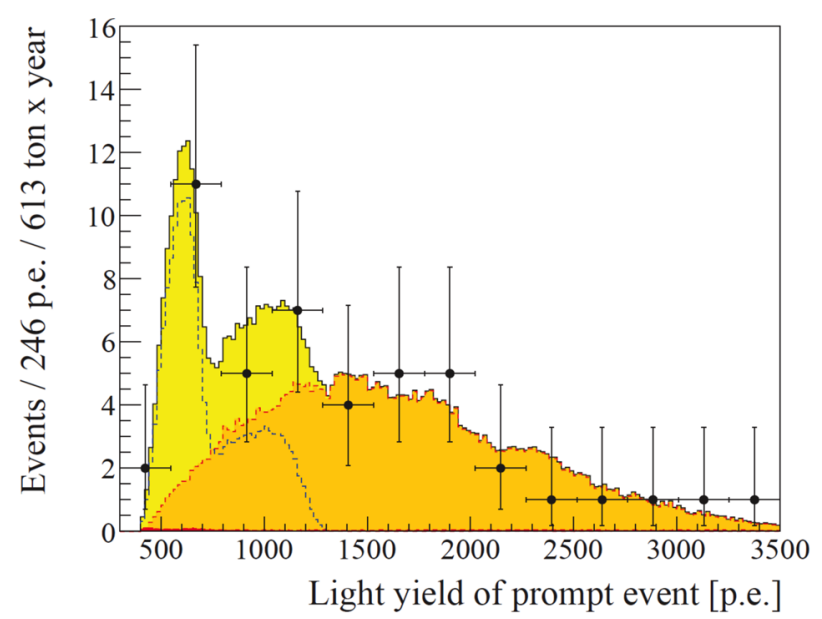

Figure 3. Light yield spectrum of the 46 prompt (positron in Equation (4)) anti-neutrino candidates and the best fit. The yellow area isolates the contribution of the geo- $\bar{v}_{\mathrm{e}}$ in the total signal. Dashed red line/ orange area: reactor $-\bar{v}_{\mathrm{e}}$ signal from the fit. Dashed blue line: geo- $\bar{v}_{\mathrm{e}}$ signal resulting from the fit. The contribution of other backgrounds is almost negligible and is shown by the small red filled area in the lower left part. The conversion from photoelectrons (p.e.) to energy is approximately 500 p.e. $/ \mathrm{MeV}$.

1 event / year $/ 10^{32}$ protons), such events correspond, respectively, to the signals $S_{\text {geo }}=(38.8 \pm 12.0)$ TNU and $S_{\text {react }}=84.5_{-16.9}^{+19.3}$ TNU. From the measured geo-neutrino signal we can infer the overall $\bar{v}_{\mathrm{e}}$ fluxes from $\mathrm{U}$ and $\mathrm{Th}$ decay, i.e. $\phi(\mathrm{U})=(2.4 \pm 0.7) \times 10^{6} \mathrm{~cm}^{-2} \mathrm{~s}^{-1}$ and $\phi(\mathrm{Th})=(2.0 \pm 0.6) \times 10^{6} \mathrm{~cm}^{-2} \mathrm{~s}^{-1}$. From the log-likelihood profile, the null geo-neutrino hypothesis has a probability of $6 \times 10^{-6}$, which is, therefore, the significance of the present geo-neutrino detection. The signal from the reactors is in full agreement with the expectations of $(33.3 \pm 2.4)$ events, in the presence of neutrino oscillations.

\section{The BSE models}

The bulk composition of the silicate Earth, the socalled bulk silicate Earth (BSE) models, describes the composition of the primitive mantle, i.e. the Earth composition after the core separation and before the crust-mantle differentiation. The estimates of the composition of the present-day mantle can be derived as a difference between the mass abundances predicted by the BSE models in the primitive mantle and those observed in the present crust. In this way, the predictions of the $U$ and Th mass abundances in the mantle can be done, which are then critical in calculating the geo-neutrino signal.

The most recent BSE-models classification is that of Šrámek et al. [2013], which distinguishes among Geochemical BSE models, Cosmochemical BSE models and Geodynamical BSE models.

The Earth's surface heat flux is estimated based on the measurements of temperature gradients along sev- eral thousands of drill holes along the globe. The most recent elaboration of these data leads to evaluating such a flux equal to $47 \pm 2 \mathrm{TW}$, consistent with the prediction in Davies and Davies [2010] and Jaupart et al. [2007]. The relative contribution of the radiogenic heat from radioactive decays to this flux (the so called Urey ratio) is not known and this is the key information which can be pinned down by the geo-neutrino measurements.

The geochemical, cosmochemical, and geodynamical models predict the radiogenic heat of $20 \pm 4,11 \pm 2$, $33 \pm 3$ TW and the corresponding Urey ratios of about $0.3,0.1$, and 0.6 , respectively. The heat producing elements (HPE) predicted by these models are distributed in the crust and in the mantle. The crustal radiogenic power was recently evaluated in Huang et al. [2013] as $6.8 /+1.4 /-1.1$ TW. By subtracting this contribution from the total radiogenic heat predicted by different BSE models, the mantle radiogenic power driving the convection and plate tectonics can be as little as $3 \mathrm{TW}$ and as much as 23 TW. The determination of this mantle contribution is therefore one of the main goals and potentials of geo-neutrino investigation.

\section{Geological implications}

The careful evaluation of the contribution of the local crust (LOC) to the total geo-neutrino signal, based on the local 3D geology around the LNGS laboratory, has been reported in Coltorti et al. [2011] as $S_{\text {geo }}(\mathrm{LOC})=$ $(9.7 \pm 1.3)$ TNU. On the other hand, the contribution from the rest of the crust (ROC), based on the recent calculation by Huang et al. [2013], results in the estimate of the geo-neutrino signal from the crust (LOC+ROC) equal to $S_{\text {geo }}$ (Crust) $=(23.4 \pm 2.8)$ TNU. Subtracting such estimated crustal contribution from the measured Borexino geo-neutrino rate, we can infer the contribution of the mantle, $S_{\text {geo }}($ Mantle $)=(15.4 \pm 12.3)$ TNU.

As we already stressed, the Earth releases radiogenic heat, $H_{g e e}$, together with geo-neutrinos in a well fixed ratio; however, the observed geoneutrino signal depends both on the abundances of the individual radioactive elements and on their distribution inside the Earth. The extraction of the radiogenic heat power from a measured $S_{\text {geo }}$ signal is, therefore, model dependent. The calculation of the expected $S_{\text {geo }}(\mathrm{U}+\mathrm{Th})$ as a function of the radiogenic heat produced by $U$ and Th, $H_{\text {geo }}(\mathrm{U}+\mathrm{Th})$ is reported in Figure 4, and it is compared to the Borexino results.

The allowed region between the red and blue lines in the plane $S_{\text {geo }}(\mathrm{U}+\mathrm{Th})$ and $H_{\text {geo }}(\mathrm{U}+\mathrm{Th})$ represents models which are consistent both with geochemical and geophysical data. For each total mass of $U$ and fixed $\mathrm{Th} / \mathrm{U}$ ratio, the maximal geo-neutrino signal (red line) can be obtained by maximizing the radiogenic material 
in the crust and allowing uniform distribution in the mantle. Similarly, the minimal signal (blue line) is obtained for the minimal radiogenic mass in the crust with the rest concentrated in a thin layer at the bottom of the mantle. Note that the maximum signal is obtained with a uniform distribution and not with the specular situation of a thin concentration layer at the top of the mantle, because the crust originated from the mantle itself, removing from it during its formation the $\mathrm{U}$ and Th elements.

The expected signal from the crust is taken from Table V of Fiorentini et al. [2012]. We have chosen as a reference the BSE model from McDonough and Sun [1995], predicting that the silicate Earth contains $\mathrm{m}(\mathrm{U})=$ $(0.8 \pm 0.1) \times 10^{17} \mathrm{~kg}$, with mass ratios $\mathrm{Th} / \mathrm{U}=3.9$ and $\mathrm{K} / \mathrm{U}=12,000$. The green region is allowed by this BSE model. The arrow "Min" indicates the contribution of the crust only. The arrow for the fully radiogenic model indicates the value of $39.3 \mathrm{TW}$ : it assumes that the total Earth surface heat flux of $(47 \pm 2)$ TW [Davies and Davies 2010] is completely due to radiogenic heat from $\mathrm{U}$, Th, and $\mathrm{K}$. Taking the relative proportions from the BSE of McDonough and Sun [1995], we get that, in a fully radiogenic Earth, $\mathrm{U}$, Th, and $\mathrm{K}$ would produce 19.1, 20.2, and 7.7 TW, respectively.

The circumstance that in Figure 4 the $+/-1 \alpha$ band of the Borexino result contains almost completely the portion of plane between the maximal and minimal line indicates that at this stage such a result does not restrict the choice among different BSE models.

We have performed another unbinned maximal likelihood fit of our 46 candidates, in which the individual contributions from the ${ }^{238} \mathrm{U}$ and ${ }^{232} \mathrm{Th}$ chains were fitted individually. In this case the best fit values are $\mathrm{N}_{\mathrm{Th}}=(3.9 \pm 4.7)$ events and $\mathrm{N}_{\mathrm{U}}=(9.8 \pm 7.2)$ events, corresponding to $\mathrm{S}_{\mathrm{Th}}=(10.6 \pm 12.7) \mathrm{TNU}$ and $\mathrm{S}_{\mathrm{U}}=$ $(26.5 \pm 19.5) \mathrm{TNU}$, or as $\bar{v}_{\mathrm{e}}$ fluxes (above $\left.0 \mathrm{MeV}\right) \phi(\mathrm{Th})=$ $(2.6 \pm 3.1) \times 10^{6} \mathrm{~cm}^{-2} \mathrm{~s}^{-1}$ and $(\mathrm{U})=(2.1 \pm 1.5) \times 10^{6}$ $\mathrm{cm}^{-2} \mathrm{~s}^{-1}$.

Although our data is compatible, within $1 \sigma$, either with only ${ }^{238} \mathrm{U}$ signal (and $\mathrm{S}_{\mathrm{Th}}=0$ ), or only ${ }^{232} \mathrm{Th}$ signal (and $S_{U}=0$ ), we note that the best fit of the Th/U ratio is in very good agreement with the chondritic value.

A geo-reactor with thermal power $<30 \mathrm{TW}$ and ${ }^{235} \mathrm{U}:{ }^{238} \mathrm{U}=0.76: 0.23$ composition was suggested by Herndon and Edgerley [2005]. It is assumed to be confined in the central part of the Earth's core within the radius of about $4 \mathrm{~km}$ [Herndon 1996]. We have produced MC spectra of the expected geo-reactor anti-neutrino. In a similar unbinned maximal likelihood fit of our 46 anti-neutrino candidates we have added another fit component, $N_{\text {geo-react }}$, while constraining $N_{\text {react }}$ to the expected value of $(33.3 \pm 2.4)$ events. All other fit de-

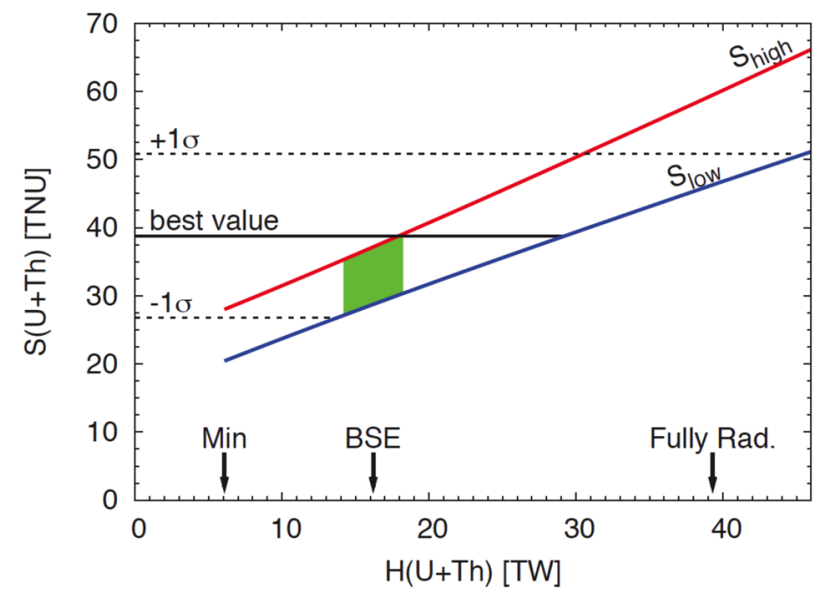

Figure 4. The signal $S_{\mathrm{U}+\mathrm{Th}}$ from $\mathrm{U}$ and Th geo-neutrinos as a function of radiogenic-heat production rate $H_{\mathrm{U}+\mathrm{Th}}$ in Borexino.

tails were as above, including fixed chondritic mass $\mathrm{Th} / \mathrm{U}$ ratio: in this way, we are able to set an upper limit on the hypothetical geo-reactor power of $4.5 \mathrm{TW}$, at $95 \%$ C.L.

\section{Future perspectives}

The geo-neutrino measurement of Borexino (together with that performed by KamLAND; Araki et al. [2005]) opened a door towards a new field, by not only proving that geo-neutrinos can be detected, but also showing that a new powerful tool to study our planet is available.

In order to find definitive answers to the questions correlated to the radiogenic heat and abundances of radiogenic elements, more data is needed. Both Borexino and KamLAND will continue to take data in the near future. In addition, it would be important to construct larger volume detectors in order to increase the number of detected geo-neutrinos and so improve the precision of the flux measurement.

Results from different detector sites placed at different geological settings is a key point for understanding, for example, if the Earth mantle composition and heat distributions are homogeneous or not. Answers to questions like what is the bulk-Earth $\mathrm{U}$ versus Th ratio, (the same as in meteorites or not) can help in better understanding of the process of Earth formation and the distribution of elements in the Solar system.

A new generation of experiments using liquid scintillators is either under design or even in advanced construction process. SNO+ at Sudbury mine in Canada [Chen 2006], having 1000 tons of target, is almost ready to start data taking. The site is located on an old continental crust and the signal from reactor anti-neutrinos is about twice as that at Gran Sasso.

The main goal of the JUNO experiment in China (formerly called Daya Bay 2) [Wang 2013] is to deter- 
mine the neutrino mass hierarchy. Thanks to a very large mass of $20 \mathrm{kton}$, it would detect up to 400 geoneutrinos per year. Unfortunately, the reactor-neutrino and cosmogenic background would be the dominant sources of the systematic error.

An ambitious project to construct a 50,000 ton detector is LENA [Wurm et al. 2012], proposed for the Pyh"asalmi mine in Finland. This experiment could detect of the order of 1000 geo-neutrinos per year, therefore a few percent precision of the total flux measurement could be reached within the first few years. The individual contribution of the $U$ and Th geo-neutrino flux could be determined as well.

An interesting project of $\sim 10,000$ ton underwater experiment is HanoHano [Learned et al. 2008], planned to be placed on the oceanic crust (Hawaii). Due to the thin oceanic crust, the mantle contribution to the total geo-neutrino flux should be dominant. Therefore, this measurement would provide the most direct information about the mantle.

If all realized, these forthcoming and proposed projects, together with the currently running experiments, would be the initial building blocks of a network of sophisticated and powerful instruments, which would greatly improve the understanding of the interior of our planet.

Acknowledgements. The author wishes to thank the organizers for the invitation to contribute to such an interesting and enlightening workshop.

\section{References}

Aglietta, M., et al. (1998). Muon "depth-intensity" relation measured by the LVD underground experiment and cosmic-ray muon spectrum at sea level, Phys. Rev. D, 58, 092005.

Ahlen, S.P., et al. (1990). Study of penetrating cosmic ray muons and search for large scale anisotropies at the Gran Sasso Laboratory, Phys. Lett. B, 249, 149-156.

Alimonti, G., et al. (Borexino collaboration) (2009). The Borexino detector at the Laboratori Nazionali del Gran Sasso, Nucl. Instrum. Meth. A, 600, 568-593.

Araki, T., et al. (KamLAND collaboration) (2005). Experimental investigation of geologically produced antineutrinos with KamLAND, Nature, 436, 499-503.

Back, H., et al. (Borexino collaboration) (2012). Borexino calibrations: Hardware, Methods, and Results, J. Instrum., 7, P10018.

Belli, P., R. Bernabei, S. d'Angelo, M. P. de Pascale, L. Paoluzi, R. Santonico, N. Taborgna, N. Iucci and G. Villoresi (1989). Deep underground neutron flux measurement with large $\mathrm{BF}_{3}$ counters, Il Nuovo Cimento A, 101 (6), 959-966.

Bellini, G., et al. (Borexino collaboration) (2009). The liquid handling systems for the Borexino solar neutrino detector, Nucl. Instrum. Meth. A, 609, 58-78.

Bellini, G., et al. (Borexino collaboration) (2011). Precision Measurement of the ${ }^{7} \mathrm{Be}$ Solar Neutrino Interaction Rate in Borexino, Phys. Rev. Lett., 107, 141302.

Bellini, G., et al. (Borexino collaboration) (2013). Measurement of geo-neutrinos from 1353 days of Borexino, Phys. Lett. B, 722, 295-300.

Bellini, G., et al. (Borexino collaboration) (2014). Final results of Borexino Phase-I on low-energy solar neutrino spectroscopy, Phys. Rev. D, 89, 112007.

Chen, M.C. (2006). Geo-neutrinos in SNO+, Earth Moon Planets, 99, 221-228.

Coltorti, M., R. Boraso, F. Mantovani, M. Morsilli, G. Fiorentini, A. Riva. G. Rusciadelli, R. Tassinari, C. Tomei, G. Di Carlo and V. Chubakov (2011). U and Th content in the Central Apennines continental crust: A contribution to the determination of the geo-neutrinos flux at LNGS, Geochim. Cosmochim. Ac., 75, 2271-2294.

Davies, J.H., and D.R. Davies (2010). Earth's surface heat flux, Solid Earth, 1, 5-24.

Enomoto, S. (2005). Neutrino Geophysics and Observation of Geo-Neutrinos at KamLAND, Ph. D. Thesis, Tohoku University, Japan.

Enomoto, S. (2009). Using neutrinos to study the Earth: geo-neutrinos, In: Proceedings of the 13th International Workshop on Neutrino Telescopes (NeuTel '09, Venice, Italy, March 2009).

Fiorentini, G., G. L. Fogli, E. Lisi, F. Mantovani and A.M. Rotunno (2012). Mantle geoneutrinos in KamLAND and Borexino, Phys. Rev. D, 86, 033004.

Fogli, G.L., E. Lisi, A. Marrone, D. Montanino, A. Palazzo and A.M. Rotunno (2012). Global analysis of neutrino masses, mixings, and phases: Entering the era of leptonic CP violation searches, Phys. Rev. D, 86, 013012.

Herndon, J.M. (1996). Substructure of the inner core of the Earth, P. Natl. Acad. Sci. USA, 93, 646-648.

Herndon, J.M., and D.A. Edgerley (2005). Background for Terrestrial Antineutrino Investigations: Radionuclide Distribution, Georeactor Fission Events, and Boundary Conditions on Fission Power Production, arXiv:hep-ph/0501216.

Huang, Y., V. Chubakov, F. Mantovani, R. Rudnick and W.F. McDonough (2013). A reference Earth model for the heat-producing elements and associated geoneutrino flux, Geochem. Geophy. Geosy., 14, 2003-2029.

Jaupart, C., S. Labrosse and J.C. Mareschal (2007). Temperatures, Heat and Energy in the Mantle of the Earth, In: G. Schubert (ed.), Treatise of Geophysics, Elsevier, Amsterdam, vol. 7, 253-303. 
Learned, J.G., S.T. Dye and S. Pakvasa (2008). Hanohano: A Deep Ocean Anti-Neutrino Detector for Unique Neutrino Physics and Geophysics Studies, In: Proceedings of the Twelfth International Workshop on Neutrino Telescopes (Venice, March 2007), arXiv:08 10.4975 .

McDonough, W.F., and S.-s. Sun (1995). The composition of the Earth, Chem. Geol., 120, 223-253.

Serenelli, A.M., W.C. Haxton and C. Peña-Garay (2011). Solar Models with Accretion. I. Application to the Solar Abundance Problem, Astrophys. J., 743 (1), article id. 24, $20 \mathrm{p}$.

Šrámek, O., W.F. McDonough, E.S. Kite, V. Lekic, S.T. Dye and S. Zhong (2013). Geophysical and geochemical constraints on geoneutrino fluxes from Earth's mantle, Earth Planet. Sci. Lett., 361, 356-366.

Strumia, A., and F. Vissani (2003). Precise quasielastic neutrino/nucleon cross-section, Phys. Lett. B, 564, 42-54.

Wang, Z. (2013). Daya Bay II update, Talk given at the Neutrino Geoscience 2013 Conference, Takayama, Japan.

Wurm, M., et al. (2012). The nextgeneration liquid-scintillator neutrino observatory LENA, Astropart. Phys., $35,685-732$

${ }^{\star}$ Corresponding author: Gioacchino Ranucci,

Università di Milano, Dipartimento di Fisica, and INFN,

Milan, Italy; email: gioacchino.ranucci@mi.infn.it.

(C) 2017 by the Istituto Nazionale di Geofisica e Vulcanologia. All rights reserved. 